BULLETIN OF THE

AMERICAN MATHEMATICAL SOCIETY

Volume 80, Number 4, July 1974

\title{
BILINEAR FORMS AND CYCLIC GROUP ACTIONS
}

\author{
BY J. P. ALEXANDER, G. C. HAMRICK AND J. W. VICK ${ }^{1}$
}

Communicated by Glen Bredon, October 11, 1973

In a recent paper [2] Conner and Raymond have given an approach to the study of smooth cyclic group actions which employs rational bilinear forms. If $K^{4 n-1}=\partial B^{4 n}$ bounds a compact oriented smooth manifold, there is a symmetric nonsingular rational bilinear form on the image of $H^{2 n}(B, K ; \boldsymbol{Q}) \rightarrow H^{2 n}(B ; \boldsymbol{Q})$ which represents an element $w(B)$ in $W(\boldsymbol{Q})$, the rational Witt ring. Denoting the signature of this form by $\operatorname{sgn}(B)$ and the unit of $W(Q)$ by $\mathbf{1}$, the peripheral invariant of $K$,

$$
\operatorname{per}(K)=w(B)-\operatorname{sgn}(B) \cdot \mathbf{1},
$$

lies in the kernel of the signature homomorphism $\Phi: W(Q) \rightarrow Z$ and is independent of the choice of $B$. In [2] there is associated with any orientation preserving diffeomorphism $\left(T, M^{4 n}\right)$ of prime period $p$ on a closed manifold an element of the kernel of $\Phi$ which we denote by $q(T, M)$, an invariant of the equivariant bordism class which vanishes on fixed point free actions. Using the peripheral invariant, Conner and Raymond computed $q(T, M)$, for $p=2$ or 3 , in terms of the fixed point information. The fundamental problem posed in [2] is the extension of this result to all primes.

In this paper we give the general formula for all primes and apply it to establish relationships between the index of $M$ and the index of the fixed set. The essence of the proof is a group isomorphism between the kernel of $\Phi$ and $\oplus_{p} W\left(\boldsymbol{Z}_{p}\right)$ where $W\left(\boldsymbol{Z}_{p}\right)$ is the Witt group of the field $\boldsymbol{Z}_{p}$ and the sum ranges over all primes. Using this isomorphism, we establish a relation between the peripheral invariant and the linking form which enables us to extend the definition of $\operatorname{per}(K)$ to any closed oriented $(4 k-1)$-manifold.

1. Bilinear forms. Let $B$ Fin denote the semigroup of isomorphism classes of symmetric nonsingular bilinear forms on finite abelian groups taking values in $\boldsymbol{Q} / \boldsymbol{Z}$. Denote by $W^{s}(\boldsymbol{Z})$ the semigroup of stable equivalence classes of nondegenerate integral bilinear forms on finitely

AMS (MOS) subject classifications (1970). Primary 55C35, 57D85.

${ }^{1}$ This research was partially supported by NSF Grant GJ32269.

Copyright (c) American Mathematical Society 1974 
generated free abelian groups, where stability means that we are allowed to add the form $x^{2}$ or the form $-x^{2}$ without altering the equivalence class. Let $W^{s}(\boldsymbol{Q})$ be the corresponding group of rational bilinear forms. Kneser and Puppe [5] have shown that there is a one-to-one correspondence between $W^{s}(\boldsymbol{Z})$ and $B$ Fin (see also [3], [6], [7], [8]).

The composition $B$ Fin $\rightarrow W^{s}(Z) \rightarrow W^{s}(\boldsymbol{Q})$ is clearly onto and generates an equivalence relation $\sim$ on $B$ Fin, which we refer to as rational equivalence of finite forms. Suppose $(\lambda, G)$ is a finite form in $B$ Fin and $K \subseteq$ $H \subseteq G$ are subgroups such that

$$
H=\{x \in G \mid \lambda(x, y)=0 \text { for all } y \in K\}=K^{\perp},
$$

the annihilator of $K . \lambda$ induces a nonsingular form $\lambda^{\prime}$ on $H / K$.

1.1. TheOREM. If $G, H, K, \lambda$ and $\lambda^{\prime}$ are as given above, $(\lambda, G) \sim$ $\left(\lambda^{\prime}, H / K\right)$ in $B$ Fin. Conversely, if $(\lambda, G)$ is rationally trivial, there is a subgroup $H \subseteq G$ such that $H=H^{\perp}$.

We denote by $\mathscr{W}$ the Grothendieck group generated by $B$ Fin modulo the subgroup generated by all forms $(\lambda, G)$ such that there is a subgroup $H \subseteq G$ with $|H|^{2}=|G|$ and $\lambda(H, H)=0$.

1.2. THEOREM. If $W\left(Z_{p}\right)$ denotes the Witt group of nonsingular bilinear forms over $\boldsymbol{Z}_{p}$, the inclusion induces an isomorphism of groups, $\oplus_{p} W\left(\boldsymbol{Z}_{p}\right) \rightarrow \approx$ $\mathscr{W}$, where the sum ranges over all primes $p$.

We can summarize the above results in the following corollary [9].

\subsection{COROLlaRY. There is a sequence of group isomorphisms}

$$
W^{s}(\boldsymbol{Q}) \approx \mathscr{W} \approx \bigoplus_{p} W\left(\boldsymbol{Z}_{p}\right)
$$

and since $W^{s}(\boldsymbol{Q})$ may be identified with the kernel of the signature homomorphism $\Phi: W(\boldsymbol{Q}) \rightarrow(Z)$, there is an isomorphism of groups (but not of rings)

$$
W(\boldsymbol{Q}) \approx W(\boldsymbol{R}) \oplus\left(\bigoplus_{p} W\left(\boldsymbol{Z}_{p}\right)\right)
$$

A form $\lambda: Z_{p} \times Z_{p} \rightarrow Q / Z$ with $\lambda(1,1)=b / p$ is completely determined up to isomorphism by $[b] \in Z_{p}^{*} / Z_{p}^{* *}$, the multiplicative group of units modulo squares. Denote the form $\lambda(1,1)=b / p$ by $\langle b\rangle_{p}$. As abelian groups we have for $p \equiv 3(\bmod 4), W\left(Z_{p}\right) \approx Z_{4}$ generated by $\langle 1\rangle_{p}$; for $p \equiv 1(\bmod 4)$, $W\left(Z_{p}\right) \approx Z_{2} \oplus Z_{2}$ generated by $\langle 1\rangle_{p}$ and $\langle a\rangle_{p}$ where $a$ is not a square $\bmod p$, and $W\left(Z_{2}\right) \approx Z_{2}$ generated by $\langle 1\rangle_{2}$. The integral form corresponding to $\langle 1\rangle_{p}$ is the $1 \times 1$ matrix $(p)$. There is a concise algorithm for constructing the matrix for the integral form corresponding to $\langle a\rangle_{p}$. 
2. Prime order actions. Let $M^{4 n-1}$ be a closed oriented smooth manifold and let $G$ be the torsion subgroup of $H^{2 n}(M ; Z)$. Recall the definition of the linking form $\lambda$ on $M$ : If $x, y \in G$ then $x=\beta(z)$ for some $z \in$ $H^{2 n-1}(M ; Q / Z)$ where $\beta$ is the Bockstein homomorphism. Then $\lambda(x, y)=$ $\langle z \cup y,[M]\rangle$ in $Q / Z$ defines a nonsingular bilinear pairing on $G$. Denote the corresponding element of $\mathscr{W}$ by $\lambda(M)$.

Suppose that $M^{4 n-1}=\partial B^{4 n}$ where $B^{4 n}$ is a compact oriented smooth manifold. For $i: M \rightarrow B$ the inclusion map, define

$$
\begin{aligned}
& H=\left\{x \in G \mid x=i^{*}(\xi) \text { for some } \xi \in H^{2 n}(B ; Z)\right\}, \text { and } \\
& K=\left\{x \in G \mid x=i^{*}(\xi) \text { for some } \xi \in \text { Tor } H^{2 n}(B ; Z)\right\} .
\end{aligned}
$$

Then $K$ is isomorphic to $G / H$. Note that a necessary condition for $B^{4 n}$ to be a rational disk is that $|G|$ be a square, since in this case $H=K$. This gives information on a question posed in [1].

2.1. COROLlaRY. If $T$ is a smooth diffeomorphism of prime period on $S^{2 k-1}$ with fixed set $M^{4 n-1}$ such that the order of $\operatorname{Tor}\left(H^{2 n}(M ; Z)\right)$ is not a square, then $T$ cannot be smoothly extended to $D^{2 k}$.

2.2. Lemma. Under the linking form $(\lambda, G), H=\{x \in G \mid \lambda(x, y)=$ 0 for all $y \in K\}=K^{\perp}$.

2.3. THEOREM. If $M^{4 n-1}=\partial B^{4 n}$ as above, then under the isomorphism $W^{s}(\boldsymbol{Q}) \approx \mathscr{W}$

$$
\operatorname{per}(M)=-\lambda(M)
$$

By taking this equation as the definition, the peripheral invariant may be extended to all closed oriented ( $4 n-1)$-manifolds (in fact, using techniques analogous to those for the index, it can be extended to compact manifolds with boundary).

2.4. Corollary. $\operatorname{per}(M)$ is defined for all closed oriented $(4 n-1)$ manifolds and is an invariant of the oriented homotopy type of $M$.

2.5. COROLlaRY. If $T$ is a smooth diffeomorphism of prime period on $S^{2 k-1}$ with fixed set $M^{4 n-1}$ having $\operatorname{per}(M) \neq 0$, then $T$ cannot be smoothly extended to $D^{2 k}$.

The lens spaces give an interesting set of examples for examining the peripheral invariant as well as for applications. The quotient of the action of $Z_{p}$ on $S^{4 n-1}$ given by $T\left(z_{1}, \cdots, z_{2 n}\right)=\left(\alpha^{r_{1}} z_{1}, \cdots, \alpha^{r_{2 n}} z_{2 n}\right)$, where $\alpha=e^{2 \pi i / p}$ and $\left(r_{j}, p\right)=1$, gives the lens space $L^{4 n-1}\left(p ; r_{1}, \cdots, r_{2 n}\right)$. For each $j$ choose an integer $l_{j}$ with $l_{j} \cdot r_{j}=1 \bmod p$. Let $l=l_{1} \cdot l_{2} \cdot \cdots \cdot l_{2 n}$.

2.6. Proposition. The linking form on this lens space is given by $\lambda\left(L^{4 n-1}\left(p ; r_{1}, \cdots, r_{2 n}\right)\right)=\langle l\rangle_{p}$. 
Conner and Raymond [2] have defined an invariant for smooth periodic group actions that fits nicely into this setting. Let $\left(T, M^{4 n}\right)$ be an orientation preserving diffeomorphism of odd prime period $p$ on a closed manifold. There is a symmetric, nonsingular bilinear form on $H^{2 n}(M ; Q)$ given by $f(x, y)=p \cdot\langle x \cup y,[M]\rangle \in \boldsymbol{Q}$. The restriction of $f$ to the fixed vectors defines an element $w(T, M) \in W(\boldsymbol{Q})$, whose signature we write as $\operatorname{sgn}(M / T)$. The invariant defined in [2] which we have denoted by $q(T, M)$ is defined by

$$
q(T, M)=w(T, M)-\operatorname{sgn}(M / T) \cdot \mathbf{1} .
$$

Since this lies in the kernel of $\Phi: W(\boldsymbol{Q}) \rightarrow \boldsymbol{Z}$, we view it as an element of $\mathscr{W}$. One of the principal results of [2] is the determination of this invariant for $p=2$ or 3 .

2.7. THEOREM (CONNER AND RAYMOND [2]). If $p=3$, or if $p=2$ and $T$ is weakly complex, then $q(T, M)=\operatorname{sgn}(F) \cdot\langle 1\rangle_{p}$ where $F$ is the fixed set.

Let $N$ be an equivariant tubular neighborhood of $F$ in $M$. The relationship between this invariant and the peripheral invariant may be stated [2] as

$$
q(T, M)=p \otimes w(N)-\operatorname{sgn}(N) \cdot \mathbf{1}-\operatorname{per}(\partial N / T)
$$

where tensoring a rational form with $p$ corresponds to multiplying each entry in its matrix by $p$.

Now suppose that $F_{0}^{2 k}$ is a component of the fixed set and $S^{2 m-1} \rightarrow \partial N_{0} \rightarrow$ $F_{0}^{2 k}$ is the equivariant sphere bundle over $F_{0}$. The quotient under $T$ is the lens space bundle $L_{0}^{2 m-1} \rightarrow \partial N_{0} / T \rightarrow F_{0}^{2 k}$.

An argument involving spectral sequences shows:

2.8. THEOREM. The local fixed point information is given by

$$
p \otimes w\left(N_{0}\right)-\operatorname{sgn}\left(N_{0}\right) \cdot 1-\operatorname{per}\left(\partial N_{0} / T\right)=\operatorname{sgn}\left(F_{0}^{2 k}\right) \cdot \lambda\left(L_{0}^{2 m-1}\right) .
$$

2.9. COROLlary. (a) For $p \equiv 3(\bmod 4)$ give the normal bundle to $F$ a complex structure in which all eigenvalues are of the form $\alpha^{k}$ where $k$ is a square $\bmod p$. Then if $F$ is given the orientation consistent with the orientation of $M$,

$$
q(T, M)=\operatorname{sgn}(F) \cdot\langle 1\rangle_{p} .
$$

(b) For $p \equiv 1(\bmod 4)$ orient $F$ arbitrarily. Let $F_{1}$ be the union of those components of $F$ in which the corresponding lens space has $\lambda(L)=\langle 1\rangle_{p}$ and $F_{2}$ the union of the remaining components. Then

$$
q(T, M)=\operatorname{sgn}\left(F_{1}\right) \cdot\langle 1\rangle_{p}+\operatorname{sgn}\left(F_{2}\right) \cdot\langle a\rangle_{p} .
$$


2.10. Corollary. Suppose $(T, M)$ is as above and $T^{*}$ is the identity on $H^{2 n}(M ; Q)$. Then with $F$ oriented as in (2.9),

(a) For $p \equiv 3(\bmod 4), \operatorname{sgn}(M) \equiv \operatorname{sgn}(F) \bmod 4$.

(b) For $p \equiv 1(\bmod 4), \operatorname{sgn}(M) \equiv \operatorname{sgn}(F) \equiv \operatorname{sgn}\left(F_{1}\right) \bmod 2$.

(Note that a unimodular form of dimension less than $p$ has no isometries of order $p$.)

Related results comparing the index of $M$ to the index of $F$ have been obtained by Lowell Jones [4] using completely different methods. It may be seen by simple examples that the relation in (a) is the best possible. We have evidence that in (b) there may be a $Z_{4}$ invariant. In fact for $p=5$ an argument using the Atiyah-Singer index theorem shows that $\operatorname{sgn}(M) \equiv$ $\operatorname{sgn}(F) \bmod 4$.

\section{REFERENCES}

1. J. P. Alexander, G. C. Hamrick and J. W. Vick, Involutions on homotopy spheres, (1973) (preprint).

2. P. E. Conner and F. Raymond, A quadratic form in the quotient of a periodic map, (1973) (preprint).

3. A. Durfee, Ph.D. Thesis, Cornell University, lthaca, N.Y., 1971.

4. L. Jones, Combinatorial symmetries of the disk (preprint).

5. M. Kneser and D. Puppe, Quadratische Formen und Verschlingungsinvarianten von Knoten, Math. Z. 58 (1953), 376-384. MR 15, 100.

6. C. T. C. Wall, Quadratic forms on finite groups, and related topics, Topology 2 (1964), 281-298. MR 28 \#133.

7. - Quadratic forms on finite groups. II, Bull. London Math. Soc. 4 (1972), $156-160$.

8. Wilkins, Ph.D. Thesis, University of Liverpool, 1971.

9. D. Husemoller and J. W. Milnor, Symmetric bilinear forms, Ergebnisse der Mathematik und ihrer Grenzgebiete, 73 (1973).

Department of Mathematics, University of Texas, Austin, TeXas 78712 